\title{
Systemic characterization of the SLC family genes reveals SLC26A6 as a novel oncogene in hepatocellular carcinoma
}

\author{
Jianzhong Cao, Penghui Wang, Jiemin Chen, Xiaodong He \\ Department of General Surgery, Peking Union Medical College Hospital, Chinese Academy of Medical Sciences \& Peking Union Medical College, \\ Beijing, China \\ Contributions: (I) Conception and design: X He, J Cao; (II) Administrative support: X He; (III) Collection and assembly of data: J Cao, P Wang, J \\ Chen; (IV) Data analysis and interpretation: J Cao; (V) Manuscript writing: All authors; (VI) Final approval of manuscript: All authors. \\ Correspondence to: Xiaodong He. Department of General Surgery, Peking Union Medical College Hospital, Chinese Academy of Medical Sciences \& \\ Peking Union Medical College, No. 1 Shuaifuyuan, Beijing 100730, China. Email: hexdpumch@sina.com.
}

\begin{abstract}
Background: Solute carrier (SLC) transporters play important roles in various physiological and pathological processes, such as cellular uptake of nutrients, cellular metabolism, tumor initiation and chemotherapy resistance. However, little is known about the comprehensive expression profile of SLC genes in human cancers, especially in human hepatocellular carcinoma (HCC).

Methods: Comprehensive analysis was performed using the TCGA dataset to evaluate the expression profile and clinical significance of SLC family genes in HCC. Real-time PCR and immunohistochemistry (IHC) assays were conducted to validate the expression of solute carrier family 26 member 6 (SLC26A6) in clinical HCC tissues. Cell Counting Kit-8 (CCK8), colony formation and subcutaneous xenograft tumorigenesis assays were carried out to explore the functional role of SLC26A6 in HCC. Bioinformatic analyses were used to predict the potential molecular mechanism of SLC26A6 in HCC.

Results: We identified 118 differentially expressed SLC genes (DESLCs) and found that there was a preferential enrichment for activated DESLCs in HCC. Clinical-relevant DESLCs identified a poor prognostic HCC subtype exhibiting unique clinicopathological and genomic profiles. SLC26A6 was overexpressed in HCC tissues both in mRNA and protein levels. Knockdown of SLC26A6 suppressed HCC tumorigenesis both in vitro and in vivo, indicating it as a promising therapeutic target. Finally, multifaceted bioinformatic analyses indicated that SLC26A6 might associate with multiple cancer-related pathways.
\end{abstract}

Conclusions: This study highlights the potential roles of SLC genes in HCC tumorigenesis, and suggested SLC26A6 as a promising therapeutic target in HCC patients.

Keywords: Solute carrier (SLC) genes; hepatocellular carcinoma (HCC); SLC26A6; prognosis

Submitted Apr 02, 2020. Accepted for publication Mar 05, 2021.

doi: $10.21037 /$ tcr-20-1751

View this article at: https://dx.doi.org/10.21037/tcr-20-1751

\section{Introduction}

Hepatocellular carcinoma (HCC) leads to more than 700,000 deaths yearly, making it to be the second leading cause of cancer-related deaths worldwide (1). Due to a low rate of early diagnosis and limited treatment options in the advanced stage, the prognosis of HCC patients remains rather poor $(2,3)$. Several systemic agents, such as sorafenib, lenvatinib and nivolumab, have been approved to be used in advanced HCC patients, leading to a survival increase of several months, which remains quite unsatisfied and falls short of what has been achieved in many other tumor types (4-6). Genetic studies have depicted the landscape of molecular aberrations associated with HCC. However, the most dominant mutational genes in HCC are not clinically actionable, such as TERT, TP53 and CTNNB1 $(7,8)$. Therefore, a better understanding of the detailed molecular mechanism underlying HCC tumorigenesis is urgently needed. 
More than $5 \%$ of all human genes are transporter-related, reflecting the biological significance of transporters and their critical roles in cell homeostasis (9). The largest group of the transporter-related genes is formed by the solute carrier (SLC) superfamily, which includes 52 families and encodes over 400 transporter proteins (10). SLC transporters are the second largest family of membrane proteins in the human genome, regulating the intake and/or efflux across cellular membranes of a wide variety of essential molecules (11). Besides the essential physiological functions, SLC transporters are associated with various pathological processes, including tumorigenesis and chemotherapy resistance $(12,13)$. The solute carrier family 12 member 5 (SLC12A5), which is an integral membrane $\mathrm{KCl}$ cotransporter, has been reported to be amplified in colorectal and ovarian cancers, serving as a putative driver gene in these cancers $(14,15)$. In HCC, SLC27A5 was found to be a tumor suppressor and suppressed TXNRD1 expression via the KEAP1/NRF2 pathway (16). Over-expression of SLC46A3 inhibited the aggressive phenotypes of HCC cells, and predicted a better prognosis for patients with HCC (17). As for SLC13A5, researchers expanded its role from facilitating hepatic energy homeostasis to promoting $\mathrm{HCC}$ cell proliferation, suggesting a potential role of SLC13A5 in HCC initiation and progression (18). However, the comprehensive expression profile and the corresponding clinical significance of the SLC transporter genes in HCC remain elusive.

Solute Carrier Family 26 Member 6 (SLC26A6) is a nonselective anion exchanger that transports anions such as oxalate, formate and sulfate (19). SLC26A6 is expressed mainly in the apical membrane of the intestines and kidneys, and is associated with intestinal oxalate secretion and the formation of kidney stones (20-22). In colorectal cancer, SLC26A6 was reported to be mutated in over $10 \%$ of the 23 colorectal cancer cell lines (23). Moreover, genetic analysis revealed that SLC26A6 mutation might associate with Hodgkin lymphoma, and SLC26A6 expression was low or absent in Hodgkin lymphoma cells (24). However, little is known about the role of SLC26A6 in tumorigenesis, especially in HCC.

In the present study, for the first time, we profiled the expression pattern of the SLC genes and identified 118 DESLCs in HCC tissues, revealing that SLC genes were closed linked to Hepatocarcinogenesis. Then, 31 clinical-relevant DESLCs classified the HCC patients into two subtypes, exhibiting distinct clinicopathological and genomic profiles. Furthermore, we demonstrated the overexpression and oncogenic role of SLC26A6 in HCC, indicating it as a promising therapeutic target. Finally, we performed bioinformatic analyses and found that SLC26A6 might associate with multiple cancer-related pathways, supporting the oncogenic role of SLC26A6 in HCC tumorigenesis. We present the following article in accordance with the STREGA reporting checklist and ARRIVE reporting checklist (available at https://dx.doi. org/10.21037/tcr-20-1751).

\section{Methods}

\section{TCGA database and bioinformatic analysis}

The gene expression (FPKM) and clinical data of HCC patients from TCGA Liver Cancer (LIHC) dataset were obtained by using UCSC Xena Browser $(25,26)$. In TCGA LIHC cohort, RNAseq data was available in 371 HCC tissues and 50 adjacent non-tumor tissues. Of the 371 HCC cases, 365 contain detailed clinical and follow-up data. The mutation and copy number variation data of HCC patients from TCGA dataset were retrieved in the cBioPortal website $(27,28)$. Gene ontology (GO) and kyoto encyclopedia of genes and genomes (KEGG) analyses were conducted to predict the function of SLC26A6 using DAVID Bioinformatics Tool (29,30). Pathway Commons and TargetScan were used to predict the interaction network of SLC26A6 $(31,32)$.

\section{Patients and tissue specimens}

Thirty paired fresh-frozen HCC and adjacent non-tumor tissue samples used in quantitative real-time PCR (qRTPCR) were randomly collected from HCC patients who underwent hepatectomy at Peking Union Medical College Hospital (PUMCH, Beijing, China) between 2010 and 2014. One hundred and sixty-five HCC, thirty-one paratumor and thirty-seven normal liver tissue samples were used in tissue microarray analysis. HCC tissue samples were consecutively chosen from 165 HCC patients who underwent hepatectomy at PUMCH between January 2010 and December 2011. Normal liver tissues were collected from patients with hepatolithiasis who were treated in the same hospital. Complete clinicopathologic and follow-up data are available for the $165 \mathrm{HCC}$ samples. All diagnoses were confirmed pathologically. The study was conducted in accordance with the Declaration of Helsinki (as revised in 2013). The study was approved by the Ethics Committee of Peking Union Medical College Hospital and informed 
consent was taken from all the patients. Experiments were performed under a project license (JS-1569) granted by the Institutional Animal Experimentation Committee of PUMCH, in compliance with national guidelines for the care and use of animals.

\section{Tissue microarray construction and immunohistochemistry}

Tissue microarrays (TMA) were constructed as presented by the previous study (33). In brief, hematoxylin and eosin (H\&E) sections were assessed and an appropriate area of tumor was marked on the corresponding paraffin block. The representative $1.5 \mathrm{~mm}$ diameter tissue cores were removed by a hollow needle and re-embedded into a recipient paraffin block at a defined position. TMA slides were pretreated at $65^{\circ} \mathrm{C}$ for 2 hours, followed by deparaffinization and antigen retrieval. Then, peroxidase activity was blocked by incubating the sections with $3 \%$ hydrogen peroxide for 10 minutes at room temperature. After blocking with $10 \%$ goat serum, the slides were incubated in primary antibodies against SLC26A6 overnight and then incubated in HRP-conjugated secondary antibodies. HRP activity was detected using the Liquid DAB+ Substrate Chromogen System (DAKO). Finally, sections were counterstained with hematoxylin and photographed.

SLC26A6 expression was evaluated using the "hybrid scoring system" (H-score) criteria based both on the percentage of positively stained cells and on the intensity of staining. In brief, the $\mathrm{H}$-score was calculated as the sum of the product of the staining intensity in tumor cells ( 0 , no staining; 1 , weak staining; 2 , moderate staining; three, intense staining) and the extent of cells showing that staining intensity $(0-100 \%)$. Therefore, the possible $\mathrm{H}$-score ranged from 0 to 300 .

\section{$R N A$ isolation, reverse transcription and quantitative real- time PCR (qRT-PCR)}

Total RNA was extracted from tissues and cells using Trizol (Invitrogen, Carlsbad, CA, USA). The reverse transcription of mRNA was performed using GoScript ${ }^{\mathrm{TM}}$ Reverse Transcription System (Promega Corporation, WI, USA) according to manufacturer's instruction. qRT-PCR was performed using LightCycler ${ }^{\circledR} 480$ real-time PCR (Roche, Basel, Switzerland). The data were normalized to GAPDH and the relative expression of target genes was calculated using the $2^{-\Delta \Delta C t}$ method. The primers for qRT-PCR were available in supplementary data (Table S1).

\section{Western blot analysis}

The total protein lysate was extracted from HCC cells using cell lysis buffer for Western blot and IP (Beyotime, Shanghai, China). Protein lysates were subjected to SDS/PAGE (10\% separation gel) and transferred onto a PVDF membrane for immunoblotting. The membranes were blocked with $5 \%$ fat-free milk for 1 hour at room temperature and then incubated with the following primary antibodies: SLC26A6 (sc-515230, 1:200; Santa Cruz Biotechnology, Texas, USA) and GAPDH (CW0100M; 1:5,000; ComWin, Beijing, China). Horseradish peroxidaseconjugated secondary antibodies were used. Signals were detected using an ECL (enhanced chemiluminescence) kit (Millipore, MA, USA).

\section{Cell lines and cell proliferation experiments}

Huh7 and PLC/PRF/5 cells were obtained from Shanghai Cell Bank, Chinese Academy of Sciences, and cultured as recommended by the supplier. Cells were transiently transfected with siRNA or negative control (NC) were transfected using Lipofectamine RNAiMAX Reagent (Invitrogen, Carlsbad, USA) following the manufacturer's protocol.

Cell Counting Kit-8 (CCK8, Dojindo, Japan) was used to detect the proliferation rate. Cells were plated at a density of $2 \times 10^{3}-5 \times 10^{3}$ cells per well in 96 -well plate and treated cells were then measured by the absorbance $(450 \mathrm{~nm})$ at the indicated time points $(0,24,48,72$ and 96 h). For colony formation assay, cells were seeded (1,000-3,000 cells/well) in 6-well plates for 7-10 days. Colonies were counted after fixed with methanol and stained with crystal violet solution.

\section{Animal experiment}

All animal procedures were performed according to the National Animal Experimentation Guidelines upon approval of the experimental protocol by the Institutional Animal Experimentation Committee of PUMCH. BALB/c nude mice (female, 6-8 weeks of age) were subcutaneously injected with Huh7 cell infected with si-SLC26A6 or NC $\left(5 \times 10^{6}\right.$ cells/mice). After $7-10$ days, the palpable tumors were intratumorally injected with si-SLC26A6 or NC twice a week for 3 weeks. For each injection, $10 \mu \mathrm{g}$ of siSLC26A6 or NC mixed with $5 \mu \mathrm{L}$ in vivo transfection reagent (Engreen, Beijing, China) in PBS solution was used according to the manufacturer's protocol. Tumor volumes 
were calculated using the following formula: $1 / 2 \times \mathrm{d}^{2} \times \mathrm{D}$.

\section{Statistical analysis}

Student's $t$ test was applied for the comparison of two groups. Survival curves were determined using the Kaplan-Meier method and compared using the log-rank test. Chi-square test was used to analyze the correlation between the clusters and the clinical or molecular features. Univariate logistic regression analysis was used to explore the associations between the DESLCs and the clinical features of HCC patients. Hierarchical cluster analysis was performed using the pheatmap function in $\mathrm{R}$ software. $\mathrm{P}$ values $<0.05$ were taken as statistically significant. All statistical analyses were conducted using the SPSS statistical software package (version 22.0), GraphPad Prism 7 and R software (V 3.6.1).

\section{Results}

\section{SLCs are predominantly upregulated in HCC compared with normal tissues}

We first analyzed the expression profiles of all 417 human SLC genes using transcriptomic data in TCGA HCC dataset, and the result of hierarchical clustering analysis presented that the 417 SLC genes could separate HCC from non-tumor tissues quite well, indicating that SLC genes were closed linked to Hepatocarcinogenesis (Figure 1A). To further identify the specific SLCs potentially involved in HCC tumorigenesis, we determined 118 deferentially expressed SLC genes (DESLCs) between HCC and non-tumor tissues (FDR $<0.05 ; \mid \log 1.5 \mathrm{FCl} \geq 1$; Mean FPKM $\geq 0.1$ ). Among the 118 DESLCs, 105 DESLCs (89\%) were upregulated in HCC compared with non-tumor tissues, indicating that there was a preferential enrichment for activated SLCs in HCC (Figure 1B).

To obtain an insight into the potential mechanisms responsible for the dysregulated SLCs, we evaluated the genetic alterations of the 118 DESLCs using genomic data of TCGA HCC dataset. We analyzed 366 HCC samples with exome sequencing and copy-number alterations (CNV) data available in cBioPortal platform, finding that amplification was the predominant form of genetic alterations of DESLCs in HCC (Table S2; Figure 1C), which was consistent with our above findings that SLCs tended to be up-regulated instead of down-regulated in HCC tissues. Moreover, further analyses demonstrated that the amplifications indeed led to the significantly increased expression levels in the majority of the $\mathrm{CNV}$-enriched DESLCs (Figure 1D).

Above all, these data indicated that SLC genes were preferentially up-regulated in HCC tissues, which might be at least partly attributed to the genetic amplification.

\section{Clinical-relevant DESLCs identifies a poor prognostic HCC subtype exhibiting unique clinicopathological and genomic profiles}

To determine the clinical-relevant DESLCs in HCC, we compared the expression of DESLCs with the critical clinicopathological features, including TNM stage, tumor grade, vascular invasion status and AFP value. Utilizing the TCGA HCC dataset, we found that 31 DESLCs were significantly associated with at least 3 out of the 4 aforementioned clinicopathological features (Table S3). Then, we conducted a hierarchical clustering analysis in HCC patients from TCGA dataset using the 31 clinicalrelevant DESLCs, resulting in two major clusters (cluster 1 and 2) (Figure 2A). We further analyzed the association between the cluster and the clinical or molecular features in HCC using the TCGA HCC datasets, finding that cluster 2 was significantly associated with TNM stage $(\mathrm{P}=0.001)$, tumor grade $(\mathrm{P}=0.0004)$, vascular invasion $(\mathrm{P}=0.002)$, AFP level $(\mathrm{P}=0.0001)$, TP53 mutation $(\mathrm{P}=0.027)$ and CTNNB1 mutation $(\mathrm{P}=0.012)$ (Figure $2 A$, Table $\mathrm{S} 4)$. Moreover, we explored the prognostic significance of the cluster in HCC patients, and revealed that cluster 2 showed a clear tendency to be associated with worse clinical outcomes, in terms of overall survival (Figure $2 B$ ) and relapse-free survival (Figure 2C). Taken together, these data demonstrated that the 31 clinical-relevant DESLCs-based classification of HCC was indeed of significant clinical significance.

\section{SLC26A6 is overexpressed in HCC tissues and correlates with poor survival}

In the above-mentioned TCGA RNA-seq data analysis, we identified SLC26A6 as the most significantly deregulated SLC gene (Figure $3 A$ ), which was also included in the clinical-relevant DESLCs. To validate the RNA-seq data experimentally, we performed qRT-PCR in clinical HCC samples, and the result showed that SLC26A6 was indeed up-regulated in HCC tissues compared with paratumor tissues (Figure 3B). Furthermore, we conducted IHC analysis in HCC tissue microarrays to investigate 
A

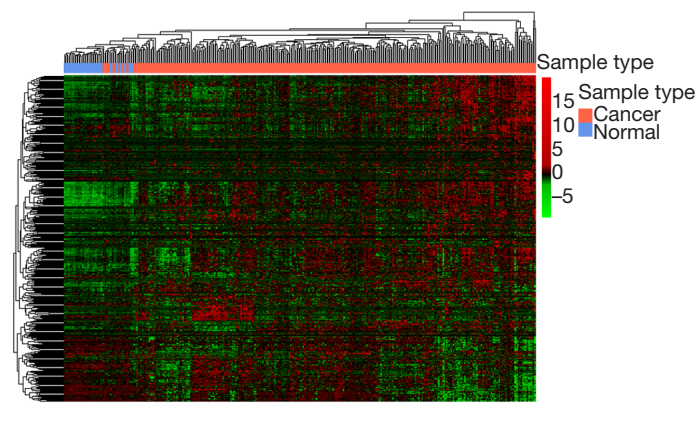

C

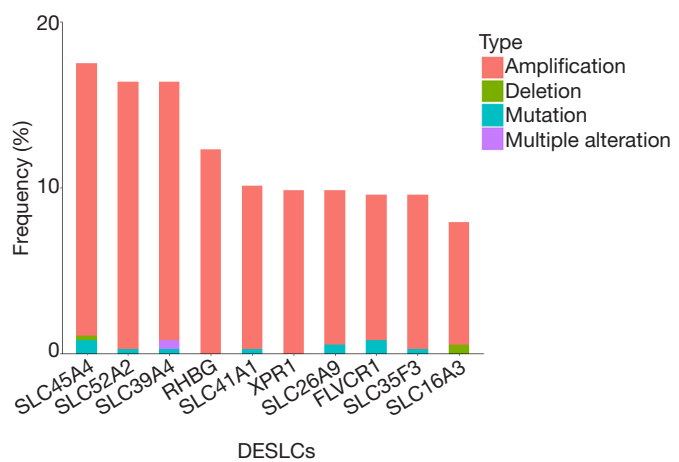

B

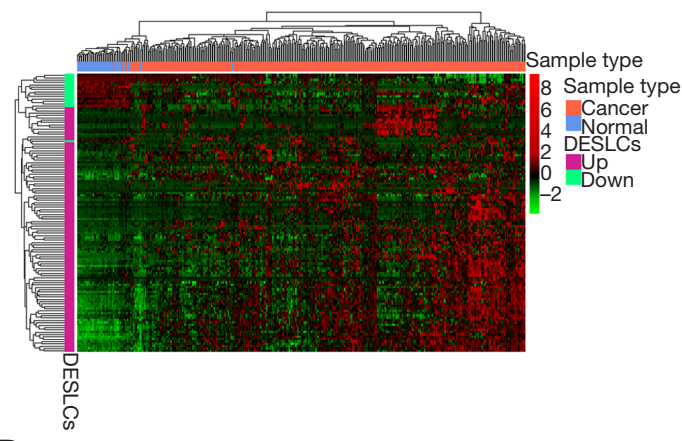

D

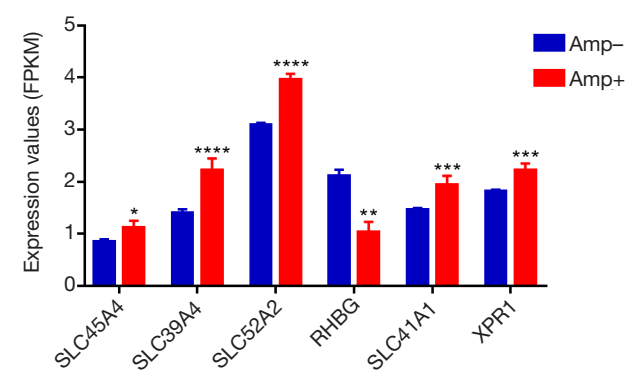

Figure 1 SLCs are predominantly upregulated in HCC compared with normal tissues. (A) Heatmap of the 417 SLC family genes expression in HCC and normal tissues from TCGA database. (B) Heatmap of the 118 DESLCs in HCC and normal tissues from TCGA database. (C) The frequency of genetic alterations (mutation, deletion, amplification or multiple alteration) of the 118 DESLCs in HCC patients from TCGA database. (D) Expression levels of the representative DESLCs in HCC patients with or without the corresponding gene amplification. ${ }^{* * *}, \mathrm{P}<0.0001 ;{ }^{* * *}, \mathrm{P}<0.001$; ${ }^{* *}, \mathrm{P}<0.01 ;{ }^{*}, \mathrm{P}<0.05$. SLCs, solute carriers; HCC, hepatocellular carcinoma.

SLC26A6 expression at the protein level, validating that SLC26A6 protein expression was also increased in HCC tissues compared with para-tumor or normal liver tissues (Figure 3C,D). Then, we examined the prognostic value of SLC26A6 overexpression in HCC patients. Kaplan-Meier analysis in the TCGA HCC dataset showed that HCC patients with high SLC26A6 expression at the mRNA level had significantly poorer survival compared with those with low SLC26A6 expression (median survival time, 1,229 and 2,131 days, respectively; $\mathrm{P}=0.005$; Figure $3 E$ ). To further confirm the above finding, we evaluated the prognostic significance of SLC26A6 protein expression in an independent HCC cohort from PUMCH, demonstrating that high SLC26A6 protein expression indeed identified a subgroup of HCC patients with poor survival (median survival time, 1,143 and 1,950 days, respectively, for SLC26A6-high and -low protein expression groups; $\mathrm{P}=0.038$; Figure $3 F$ ). Taken together, we concluded that
SLC26A6 was frequently up-regulated in HCC tissues and correlated with poor survival.

\section{SLC26A6 inbibiting demonstrates potent anti-tumor effects against HCC both in vitro and in vivo}

To investigate the potential biological function of SLC26A6 in HCC cells, we knocked down SLC26A6 expression in Huh7 and PLC/PRF/5 cells. The knockdown efficiency was measured by qRT-PCR and Western blotting (Figure 4A,B,C). As showed in Figure 4D,E, cck8 assays revealed that SLC26A6 silencing significantly repressed the proliferation of both Huh7 and PLC/PRF/5 cells. The similar results were obtained in colony formation assays in Huh7 (Figure 4F,G) and PLC/PRF/5 cells (Figure 4H,I). To further verify the oncogenic function of SLC26A6 in HCC in vivo, we then established the subcutaneous xenograft model in nude mice. The mice received intramural 
A

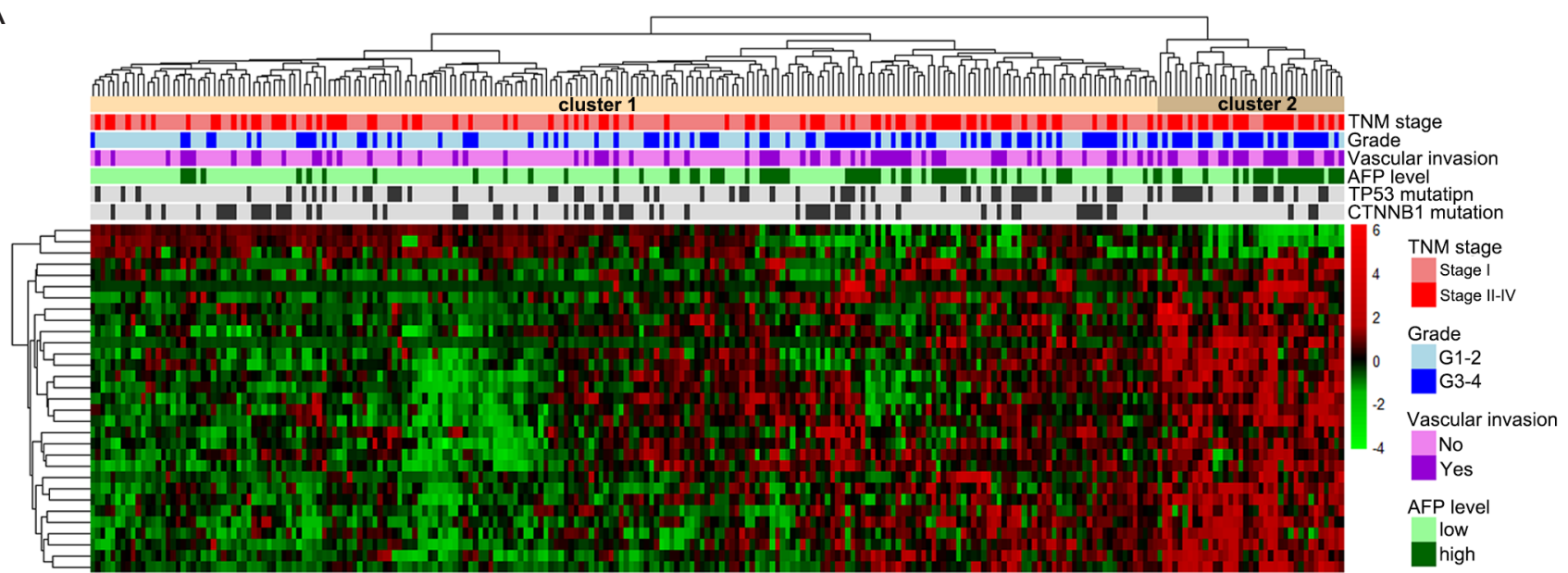

B

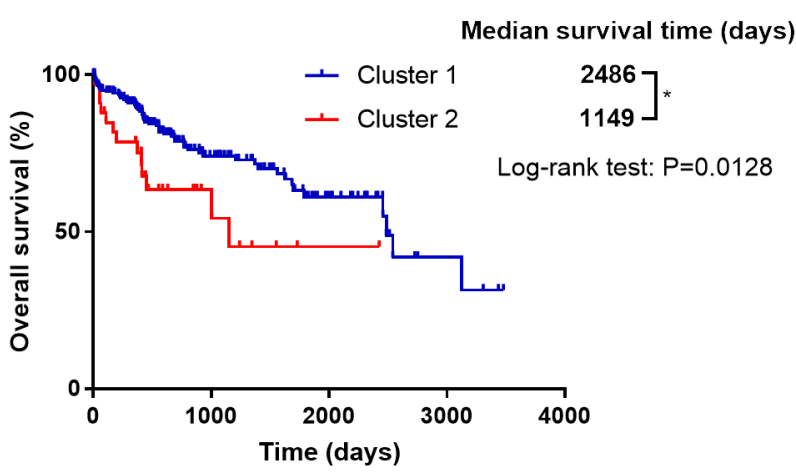

C

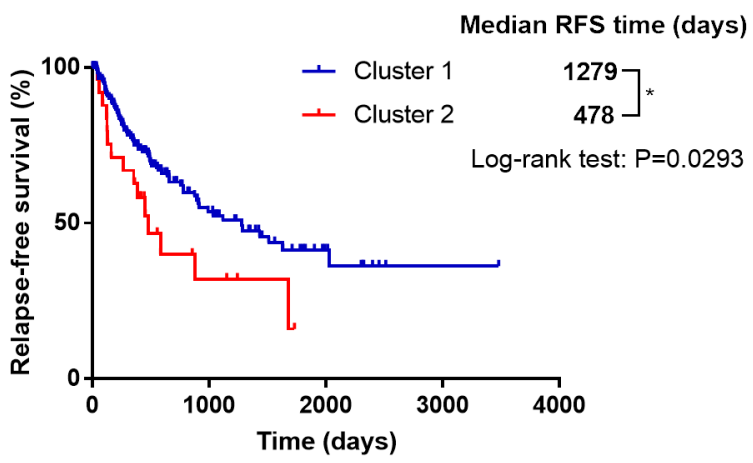

Figure 2 Clinical-relevant DESLCs identifies a poor prognostic HCC subtype exhibiting unique clinicopathological and genomic profiles. (A) Hierarchical clustering based on the 31 clinical-relevant DESLCs in HCC patients from TCGA dataset. (B,C) Kaplan-Meier curves for HCC patients in TCGA cohort between the two clusters in terms of overall survival and relapse-free survival. *, $\mathrm{P}<0.05$. HCC, hepatocellular carcinoma.

injections of si-SLC26A6 or NC, and the gross observation of tumors from both groups was shown in Figure 47. The tumor volume (Figure 4K) and weight (Figure 4L) at the end of the experiment were both markedly lower in the si-SLC26A6 group compared with NC group $(\mathrm{P}=0.0181$ and 0.0186 , respectively). Altogether, those data demonstrated that knockdown of SLC26A6 suppressed HCC tumorigenesis both in vitro and in vivo, indicating it as a promising therapeutic target in HCC.

\section{Molecular mechanisms underlying the effects of SLC26A6}

Based on the aforementioned analyses, we further investigated the potential molecular mechanism underlying
SLC26A6 overexpression in HCC pathogenesis. Utilizing cBioPortal website, we firstly analyzed the genetic alterations of SLC26A6 in HCC patients from TCGA dataset, including mutation and copy number variation. The result showed that only $1.1 \%$ of the 366 HCC patients presented genetic alterations (Figure 5A). Then, Pathway Commons used to explore the interaction network of SLC26A6, finding that 25 genes may interacted with SLC26A6 in three different ways (control its expression, binding and catalysis) (Figure 5B). Considering that miRNAs played a significant role in regulating gene expression at post-transcriptional level, we further predicted the miRNAs targeting SLC26A6 using TargetScan website, revealing that has-miR-4319, has-miR-125a-5p and has-miR-125b- 
A

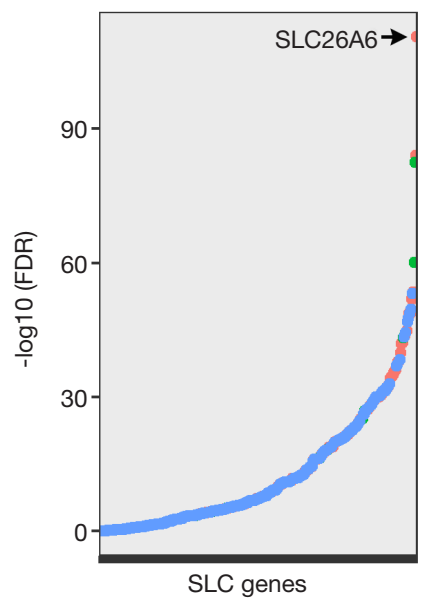

B

- Up-regulated

- Down-regulated

- Not significant
C

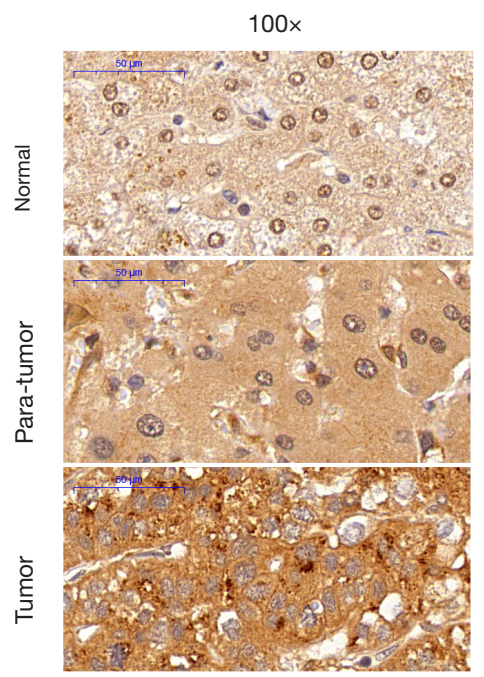

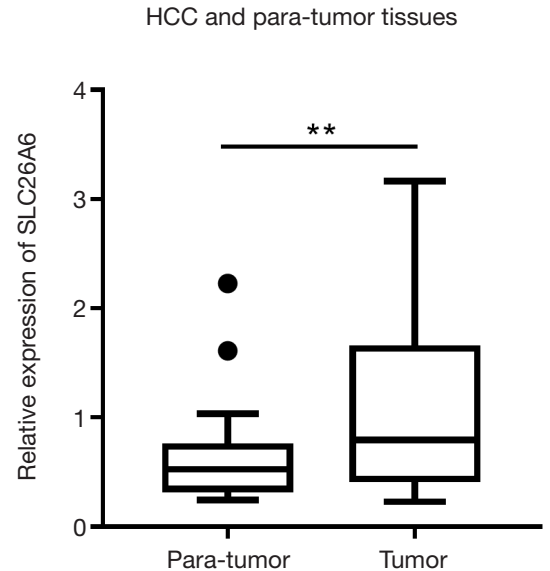

E

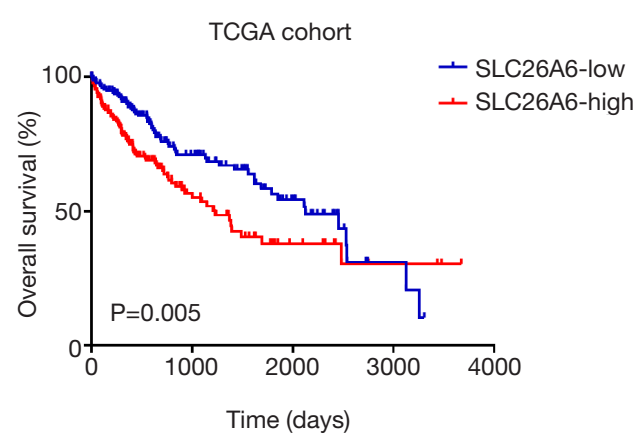

HCC tissue array

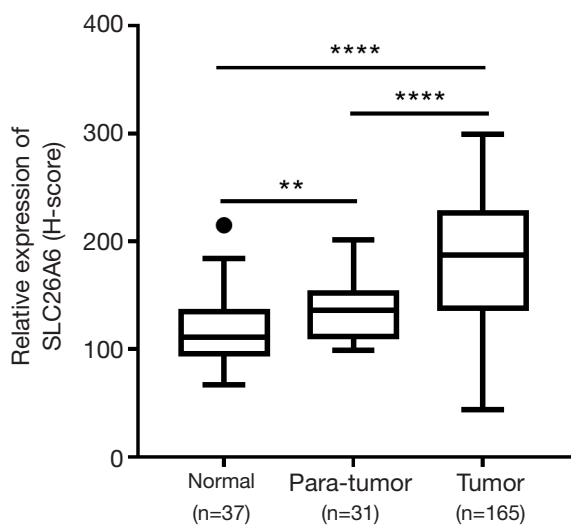

$\mathrm{F}$

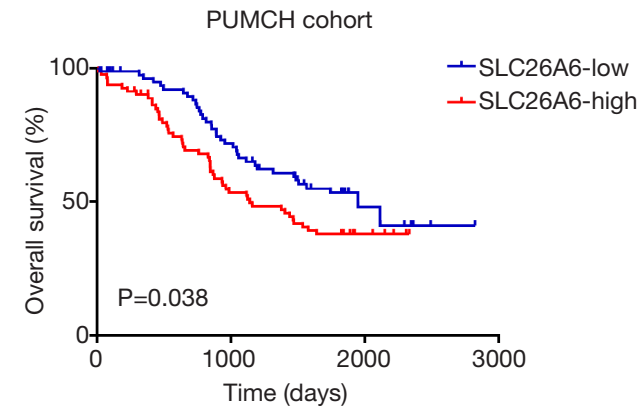

Figure 3 SLC26A6 is overexpressed in HCC tissues and correlates with poor survival. (A) Scatter plot showing the - $\log 10$ FDR values of the 417 SLC family genes when compared their expression between HCC and normal tissues from TCGA dataset. (B) Expression level of SLC26A6 in 30 paired HCC and para-tumor tissues from PUMCH cohort. (C) Representative photographs of SLC26A6 expression in TMA sections of normal liver, para-tumor and HCC tissues. (D) Comparison of H-scores of SLC26A6 between normal liver ( $\mathrm{n}=37$ ), paratumor $(\mathrm{n}=31)$ and HCC tissues $(\mathrm{n}=165)$. $(\mathrm{E}, \mathrm{F})$ Kaplan-Meier curves for HCC patients in TCGA or PUMCH cohort by SLC26A6 mRNA or protein expression. ${ }^{* * * *}, \mathrm{P}<0.0001$; **, $\mathrm{P}<0.01$. HCC, hepatocellular carcinoma. 

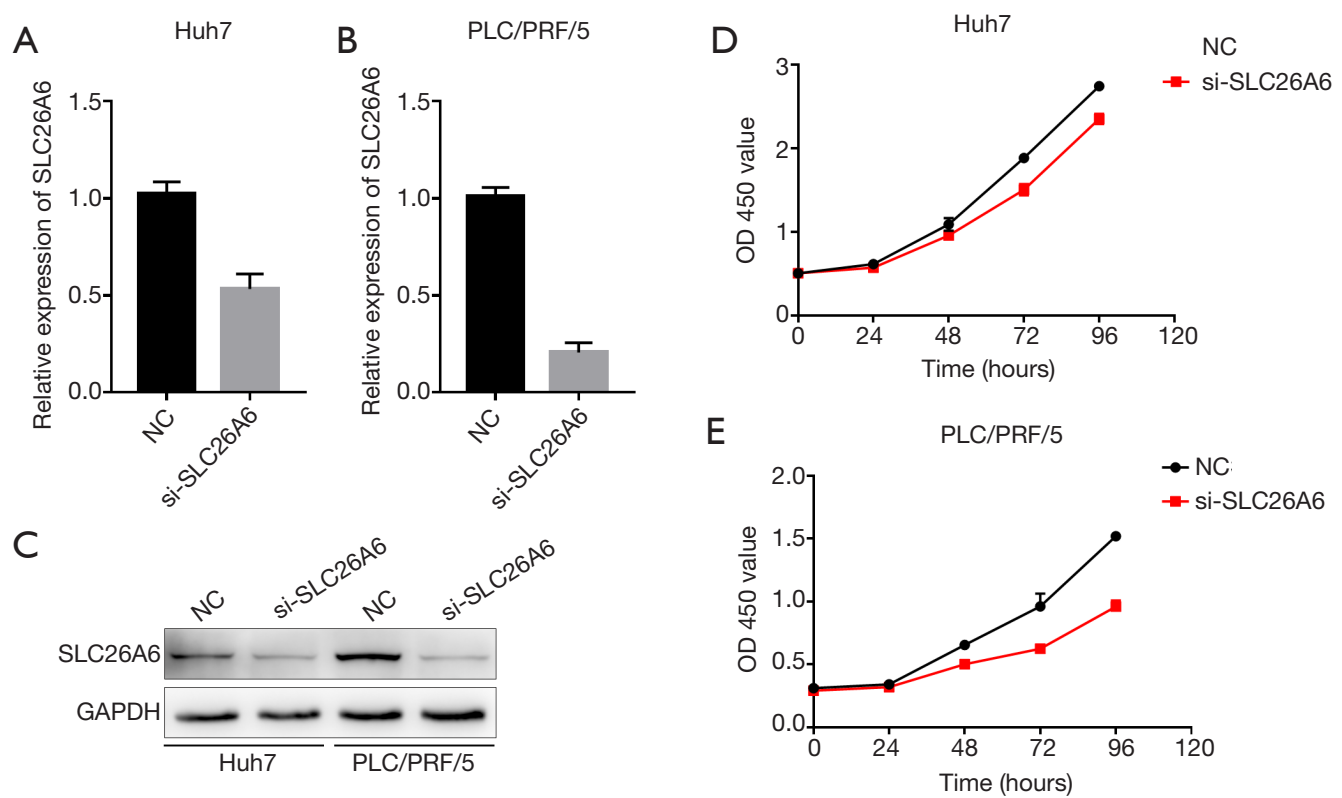

F
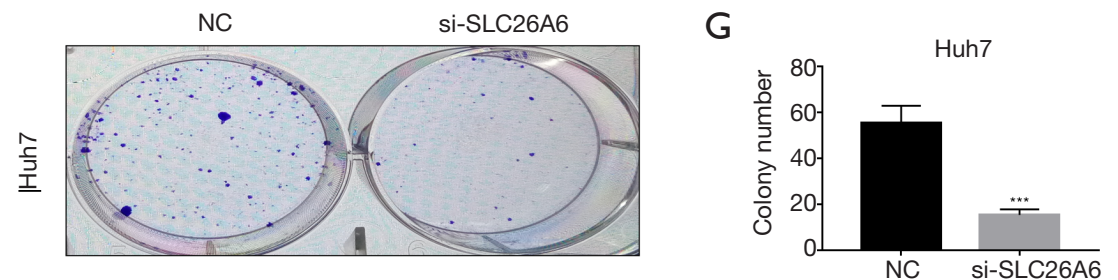

$\mathrm{H}$

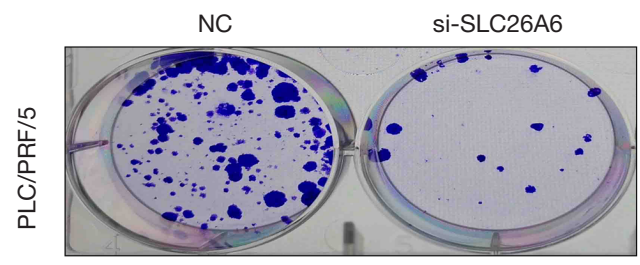

I

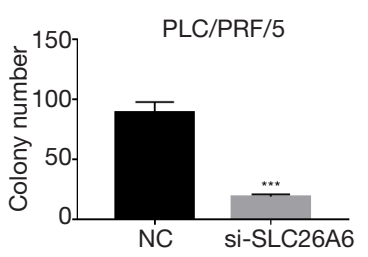

J

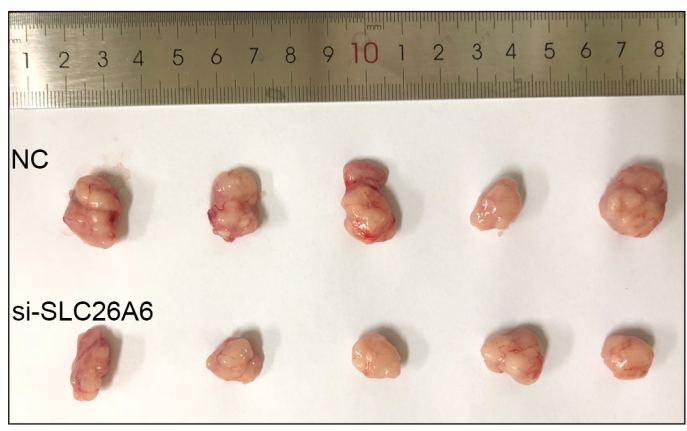

$\mathrm{K}$

L
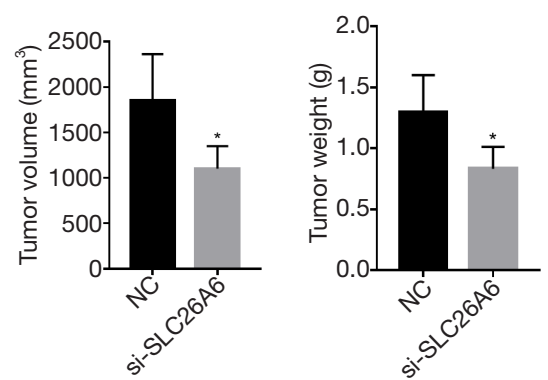

Figure 4 SLC26A6 inhibiting demonstrates potent anti-tumor effects against HCC both in vitro and in vivo. (A,B) Transfection effects of siRNA targeting SLC26A6 or negative control (NC) in Huh7 and PLC/PRF/5 cells were confirmed by qRT-PCR. (C) Transfection effects of siRNA targeting SLC26A6 or NC in Huh7 and PLC/PRF/5 cells were confirmed by western blot. (D,E) CCK8 assays showing the effects of SLC26A6 knockdown on the proliferation of Huh7 and PLC/PRF/5 cells. (F,G,H,I) Colony formation assays and the corresponding quantifications showing the effects of SLC26A6 knockdown on the proliferation of Huh7 and PLC/PRF/5 cells. Magnification in (F,H) 1×. (J) The gross observation of tumors from si-SLC26A6 and NC groups. (K,L) The tumor volume and weight of si-SLC26A6 and NC groups at the end of the experiment. ${ }^{* *}, \mathrm{P}<0.001 ;^{*}, \mathrm{P}<0.05$. HCC, hepatocellular carcinoma. 
A
SLC26A6 $1.1 \%$
Genetic alteration
1
Truncating Mutation (unknown significance)
Amplification
Deep deletion
No alterations
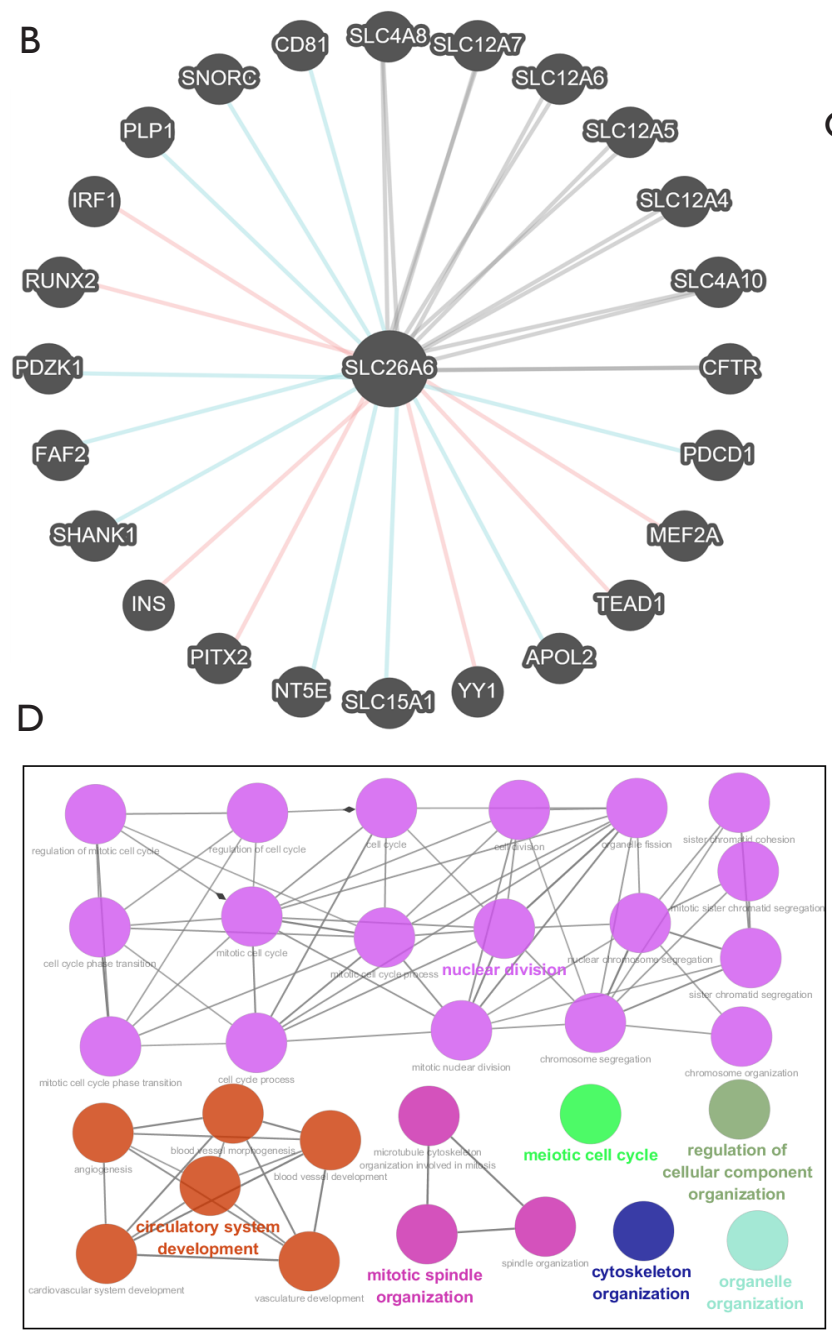

C

Predicted miRNAs targeting SLC26A6 (conserved site)

\begin{tabular}{lll}
\hline miRNA name & Position & Score \\
\hline hsa-miR-4319 & $121-128$ & -0.58 \\
hsa-miR-125a-5p & $121-128$ & -0.60 \\
hsa-miR-125b-5p & $121-128$ & -0.61 \\
\hline
\end{tabular}

E

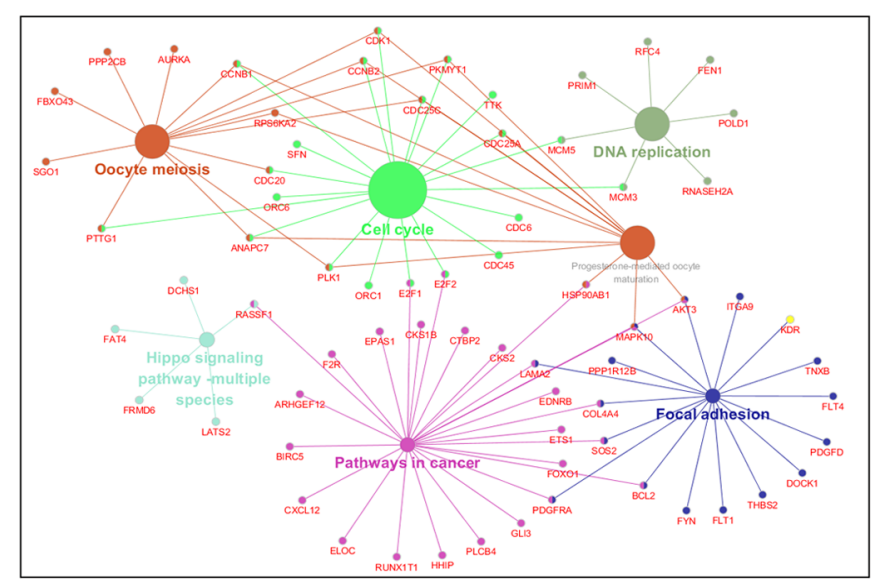

Figure 5 Molecular mechanisms underlying the effects of SLC26A6. (A) Genetic alterations of SLC26A6 in HCC patients from TCGA dataset. (B) Pathway Commons analysis showing the molecular interaction network of SLC26A6 in three different ways (control its expression, binding and catalysis). (C) MiRNAs predicted to target SLC26A6 in conserved site using Targetscan. (D) GO analysis of SLC26A6-associated genes in HCC tissues. (E) KEGG pathway analysis of SLC26A6-associated genes in HCC tissues. HCC, hepatocellular carcinoma.

$5 \mathrm{p}$ might be responsible for SLC26A6 dysregulation in HCC tumorigenesis (Figure 5C). To preliminarily explore the biological role of SLC26A6 in HCC tumorigenesis, we conducted functional enrichment analyses for the genes associated with SLC26A6 (I spearman's correlation coefficient $1 \geq 0.35$ ) using TCGA HCC RNAseq data. GO analysis demonstrated that multiple cell cycle-related biological processes were significantly enriched among the SLC26A6-associated genes in HCC (Figure 5D). Moreover, KEGG analysis further indicated that multiple cancerrelated pathways, such as Cell cycle, DNA replication, Focal adhesion, Pathways in cancer and Hippo signaling pathway, were indeed significantly enriched in SLC26A6-associated genes, supporting the oncogenic role of SLC26A6 in HCC 
tumorigenesis (Figure 5E).

\section{Discussion}

The SLC superfamily, despite its clear relevance to health and disease, is comparatively less well studied than other gene families (10). In this study, for the first time, we provide a systematic overview of the expression profile of the SLC transporter genes in HCC, demonstrating the potential involvement of SLC genes in hepatocarcinogenesis. Additionally, we then experimentally validated the dysregulation and oncogenic property of SLC26A6 in HCC, suggesting it as a potential therapeutic target in HCC patients.

Liver is metabolically active organ, and liver cancer displays a marked refractoriness to the available chemotherapy (34). Metabolic alteration is a hallmark of cancer, and commonly results in aberrant expression of SLC transporters, which linked closed to the chemoresistance (13). Consistently, in this study, we found that the 417 SLC genes could separate HCC from non-tumor tissues quite well, implying the significance of SLC family genes in hepatocarcinogenesis. We identified 118 aberrantly expressed SLC genes, and most of them were activated in HCC tissues. Many of the identified DESLCs, such as SLC39A4, RHBG, FLVCR1, SLC16A3, SLC22A1, SLC12A1, SLC29A2 and SLCO1B3 had been reported to play significant roles in HCC tumorigenesis (35-42). Other DESLCs, such as XPR1, SLC22A23 and SLC2A5, were reported to be related to the other types of cancer (43-45). Interestingly, quite a few candidate DESLCs, such as SLC45A4, SLC26A9 and SLC26A6, remain uncharacterized in tumorigenesis.

We then identified an interesting subset of DESLCs with significant clinical relevance. We found that 31 DESLCs associated with 3 out of 4 clinicopathological features (TNM stage, tumor grade, vascular invasion status and AFP value) in HCC patients, and demonstrating that these clinical-relevant DESLCs could classify HCC patients into two clusters with distinct clinicopathological and genomic profiles. SLC22A1, one of the clinicalrelevant DESLCs, have been reported to be repressed in HCC tissues. Researchers found that aberrant SLC22A1 variants, together with the decreased SLC22A1 expression, might significantly affect the response to sorafenib in HCC patients (39). Previous study related iron metabolism with HCC, and revealed that FLVCR1 expression was associated with survival, disease status, and prognosis in HCC patients (37). SLC39A4, a zinc transporter, was activated in HCC and was reported to repress apoptosis, enhance cell cycle and increase migration in HCC cells (35). However, the clinical and biological significance of SLC26A6, which was the most significantly deregulated DESLCs, remains elusive in hepatocarcinogenesis.

SLC26A6 is a typical transporter gene and mediates the exchange of pairs of anions (19). However, rather few studies relate SLC26A6 to tumorigenesis. In a previous mutational analysis carried in colorectal cell lines, SLC26A6 was found to be mutated in $>10 \%$ of the 23 colorectal cancer cell lines, suggesting that SLC26A6 might play a significant role in colorectal cancer (23). Nevertheless, in another study, protein-disrupting mutations in SLC26A6 was found in familial classical Hodgkin lymphoma (cHL) individuals, implying that SLC26A6 silencing might associate with the risk of cHL (24). In this study, we found that overexpressed SLC26A6 associated with poor survival in HCC patients, and its knockdown inhibited the malignant phenotype of HCC cells, demonstrating that SLC26A might serve as an oncogene in HCC tumorigenesis. In terms of mechanistic aspect, we performed bioinformatic analysis, predicting the underlying regulating mechanism of SLC26A6 in HCC tumorigenesis. Interestingly, it was reported that miR$125 \mathrm{a}-5 \mathrm{p}$ was a regulator of SLC26A6 in intestinal epithelial cells, which was consistent with the predicted result in our study (46). GO and KEGG analyses revealed that SLC26A6-associated genes were enriched in multiple cancer-related pathways, which further suggested the oncogenic role of SLC26A6 in HCC. However, in this study, the detailed mechanisms of the candidate SLC genes in HCC lack experimental evidence. Therefore, the future studies may focus on depicting the detailed molecular mechanisms of the candidate SLC genes in HCC tumorigenesis and developing novel drugs to target the candidate genes.

\section{Conclusions}

In summary, the present study comprehensively characterized the expression profile of SLC family genes and identified a set of clinical-relevant DESLCs with prognostic implication in HCC. Moreover, this study revealed that SLC26A6, an uncharacterized gene in tumorigenesis, might act as an oncogene in HCC and serve 
as a potential therapeutic target in HCC patients.

\section{Acknowledgments}

The authors thank Pan Liu from Peking Union Medical College (PUMC) for language polishing.

Funding: This work was supported by CAMS Initiative for Innovative Medicine (CAMS-I2M) (2017-12M-4-003) and the National Natural Science Foundation of China (81372578, to XH).

\section{Footnote}

Reporting Checklist: The authors have completed the STREGA reporting checklist and ARRIVE reporting checklist. Available at https://dx.doi.org/10.21037/tcr-201751

Data Sharing Statement: Available at https://dx.doi. org/10.21037/tcr-20-1751

Conflicts of Interest: All authors have completed the ICMJE uniform disclosure form (available at https://dx.doi. org/10.21037/tcr-20-1751). The authors have no conflicts of interest to declare.

Ethical Statement: The authors are accountable for all aspects of the work in ensuring that questions related to the accuracy or integrity of any part of the work are appropriately investigated and resolved. The study was conducted in accordance with the Declaration of Helsinki (as revised in 2013). The study was approved by the Ethics Committee of Peking Union Medical College Hospital and informed consent was taken from all the patients. Experiments were performed under a project license (JS1569) granted by the Institutional Animal Experimentation Committee of PUMCH, in compliance with national guidelines for the care and use of animals.

Open Access Statement: This is an Open Access article distributed in accordance with the Creative Commons Attribution-NonCommercial-NoDerivs 4.0 International License (CC BY-NC-ND 4.0), which permits the noncommercial replication and distribution of the article with the strict proviso that no changes or edits are made and the original work is properly cited (including links to both the formal publication through the relevant DOI and the license). See: https://creativecommons.org/licenses/by-nc-nd/4.0/.

\section{References}

1. Torre LA, Bray F, Siegel RL, et al. Global cancer statistics, 2012. CA Cancer J Clin 2015;65:87-108.

2. Bruix J, Gores GJ, Mazzaferro V. Hepatocellular carcinoma: clinical frontiers and perspectives. Gut 2014;63:844-55.

3. Forner A, Reig M, Bruix J. Hepatocellular carcinoma. Lancet 2018;391:1301-14.

4. Kudo M, Finn RS, Qin S, et al. Lenvatinib versus sorafenib in first-line treatment of patients with unresectable hepatocellular carcinoma: a randomised phase 3 noninferiority trial. Lancet 2018;391:1163-73.

5. Meyer T. Treatment of advanced hepatocellular carcinoma: beyond sorafenib. Lancet Gastroenterol Hepatol 2018;3:218-20.

6. Yau T, Hsu C, Kim TY, et al. Nivolumab in advanced hepatocellular carcinoma: Sorafenib-experienced Asian cohort analysis. J Hepatol 2019;71:543-52.

7. Llovet JM, Kelley RK, Villanueva A, et al. Hepatocellular carcinoma. Nat Rev Dis Primers 2021;7:6.

8. Schulze K, Imbeaud S, Letouze E, et al. Exome sequencing of hepatocellular carcinomas identifies new mutational signatures and potential therapeutic targets. Nat Genet 2015;47:505-11.

9. Hediger MA, Romero MF, Peng JB, et al. The ABCs of solute carriers: physiological, pathological and therapeutic implications of human membrane transport proteinsIntroduction. Pflugers Arch 2004;447:465-8.

10. César-Razquin A, Snijder B, Frappier-Brinton T, et al. A Call for Systematic Research on Solute Carriers. Cell 2015;162:478-87.

11. Colas C, Ung PM, Schlessinger A. SLC Transporters: Structure, Function, and Drug Discovery. Medchemcomm 2016;7:1069-81.

12. Huang Y, Anderle P, Bussey KJ, et al. Membrane transporters and channels: role of the transportome in cancer chemosensitivity and chemoresistance. Cancer Res 2004;64:4294-301.

13. Nyquist MD, Prasad B, Mostaghel EA. Harnessing Solute Carrier Transporters for Precision Oncology. Molecules 2017;22:539.

14. Xu L, Li X, Cai M, et al. Increased expression of Solute carrier family 12 member 5 via gene amplification contributes to tumour progression and metastasis and associates with poor survival in colorectal cancer. Gut 2016;65:635-46.

15. Yang GP, He WP, Tan JF, et al. Overexpression of 
SLC12A5 is associated with tumor progression and poor survival in ovarian carcinoma. Int J Gynecol Cancer 2019;29:1280-4.

16. Gao Q, Zhang G, Zheng Y, et al. SLC27A5 deficiency activates NRF2/TXNRD1 pathway by increased lipid peroxidation in HCC. Cell Death Differ 2020;27:1086-104.

17. Zhao Q, Zheng B, Meng S, et al. Increased expression of SLC46A3 to oppose the progression of hepatocellular carcinoma and its effect on sorafenib therapy. Biomed Pharmacother 2019;114:108864.

18. Li Z, Li D, Choi EY, et al. Silencing of solute carrier family 13 member 5 disrupts energy homeostasis and inhibits proliferation of human hepatocarcinoma cells. J Biol Chem 2017;292:13890-901.

19. Alper SL, Sharma AK. The SLC26 gene family of anion transporters and channels. Mol Aspects Med 2013;34:494-515.

20. Jiang H, Pokhrel G, Chen Y, et al. High expression of SLC26A6 in the kidney may contribute to renal calcification via an SLC26A6-dependent mechanism. PeerJ 2018;6:e5192.

21. Karaica D, Breljak D, Loncar J, et al. Sex-independent expression of chloride/formate exchanger Cfex (Slc26a6) in rat pancreas, small intestine, and liver, and maledominant expression in kidneys. Arh Hig Rada Toksikol 2018;69:286-303.

22. Seidler U, Rottinghaus I, Hillesheim J, et al. Sodium and chloride absorptive defects in the small intestine in Slc26a6 null mice. Pflugers Arch 2008;455:757-66.

23. Donnard E, Asprino PF, Correa BR, et al. Mutational analysis of genes coding for cell surface proteins in colorectal cancer cell lines reveal novel altered pathways, druggable mutations and mutated epitopes for targeted therapy. Oncotarget 2014;5:9199-213.

24. Lawrie A, Han S, Sud A, et al. Combined linkage and association analysis of classical Hodgkin lymphoma. Oncotarget 2018;9:20377-85.

25. Cancer Genome Atlas Research Network; Weinstein JN, Collisson EA, et al. The Cancer Genome Atlas PanCancer analysis project. Nat Genet 2013;45:1113-20.

26. Goldman M, Craft B, Hastie M, et al. The UCSC Xena platform for public and private cancer genomics data visualization and interpretation. bioRxiv 2019:326470.

27. Cerami E, Gao J, Dogrusoz U, et al. The cBio cancer genomics portal: an open platform for exploring multidimensional cancer genomics data. Cancer Discov 2012;2:401-4.

28. Gao J, Aksoy BA, Dogrusoz U, et al. Integrative analysis of complex cancer genomics and clinical profiles using the cBioPortal. Sci Signal 2013;6:pl1.

29. Huang $W$, Sherman BT, Lempicki RA. Systematic and integrative analysis of large gene lists using DAVID bioinformatics resources. Nat Protoc 2009;4:44-57.

30. Huang W, Sherman BT, Lempicki RA. Bioinformatics enrichment tools: paths toward the comprehensive functional analysis of large gene lists. Nucleic Acids Res 2009;37:1-13

31. Agarwal V, Bell GW, Nam JW, et al. Predicting effective microRNA target sites in mammalian mRNAs. Elife 2015;4:e05005.

32. Rodchenkov I, Babur O, Luna A, et al. Pathway Commons 2019 Update: integration, analysis and exploration of pathway data. Nucleic Acids Res 2020;48:D489-D497.

33. Cao J, Wang P, Chen J, et al. PIGU overexpression adds value to TNM staging in the prognostic stratification of patients with hepatocellular carcinoma. Hum Pathol 2019;83:90-9.

34. Marin JJG, Briz O, Herraez E, et al. Molecular bases of the poor response of liver cancer to chemotherapy. Clin Res Hepatol Gastroenterol 2018;42:182-92.

35. Weaver BP, Zhang Y, Hiscox S, et al. Zip4 (Slc39a4) expression is activated in hepatocellular carcinomas and functions to repress apoptosis, enhance cell cycle and increase migration. PLoS One 2010;5:e13158.

36. Merhi A, De Mees C, Abdo R, et al. Wnt/beta-Catenin Signaling Regulates the Expression of the Ammonium Permease Gene RHBG in Human Cancer Cells. PLoS One 2015;10:e0128683.

37. Shen Y, Li X, Zhao B, et al. Iron metabolism gene expression and prognostic features of hepatocellular carcinoma. J Cell Biochem 2018;119:9178-204.

38. Wilkins LR, Brautigan DL, Wu H, et al. Cinnamic Acid Derivatives Enhance the Efficacy of Transarterial Embolization in a Rat Model of Hepatocellular Carcinoma. Cardiovasc Intervent Radiol 2017;40:430-7.

39. Herraez E, Lozano E, Macias RI, et al. Expression of SLC22A1 variants may affect the response of hepatocellular carcinoma and cholangiocarcinoma to sorafenib. Hepatology 2013;58:1065-73.

40. Teng F, Guo M, Liu F, et al. Treatment with an SLC12A1 antagonist inhibits tumorigenesis in a subset of hepatocellular carcinomas. Oncotarget 2016;7:53571-82.

41. Chen CF, Hsu EC, Lin KT, et al. Overlapping highresolution copy number alterations in cancer genomes identified putative cancer genes in hepatocellular carcinoma. Hepatology 2010;52:1690-701. 
42. Sekine S, Ogawa R, Ojima H, et al. Expression of SLCO1B3 is associated with intratumoral cholestasis and CTNNB1 mutations in hepatocellular carcinoma. Cancer Sci 2011;102:1742-7.

43. Chen WC, Li QL, Pan Q, et al. Xenotropic and polytropic retrovirus receptor 1 (XPR1) promotes progression of tongue squamous cell carcinoma (TSCC) via activation of NF-kappaB signaling. J Exp Clin Cancer Res 2019;38:167.

44. Ekizoglu S, Seven D, Ulutin T, et al. Investigation of the SLC22A23 gene in laryngeal squamous cell carcinoma.

Cite this article as: Cao J, Wang P, Chen J, He X. Systemic characterization of the SLC family genes reveals SLC26A6 as a novel oncogene in hepatocellular carcinoma. Transl Cancer Res 2021;10(6):2882-2894. doi: 10.21037/tcr-20-1751
BMC Cancer 2018;18:477.

45. Chen WL, Wang YY, Zhao A, et al. Enhanced Fructose Utilization Mediated by SLC2A5 Is a Unique Metabolic Feature of Acute Myeloid Leukemia with Therapeutic Potential. Cancer Cell 2016;30:779-91.

46. Anbazhagan AN, Priyamvada S, Borthakur A, et al. miR125a-5p: a novel regulator of SLC26A6 expression in intestinal epithelial cells. Am J Physiol Cell Physiol 2019;317:C200-C208. 


\section{Supplementary}

\begin{tabular}{ll}
\hline Primer & sequence \\
\hline SLC26AG-F & CGGAGGCGAGACTACACACA
\end{tabular}

\begin{tabular}{ll} 
SLC26AG-R ACCAAACCGGGAGGTGTG \\
GAPDH-F & GGAGCGAGATCCCTCCAAAT \\
\hline
\end{tabular}

GAPDH-R GGCTGTGGTATACTCTCATGG

Table S2 The genetic alterations in HCC patients from TCGA cohort (n=366)

\begin{tabular}{lr}
\hline Gene symbol & Number of sam \\
\hline SLC45A4 & 64 \\
SLC3944 & 60
\end{tabular}

SLC45A4
SLC39A4

SLC52A2
RHBG

SLCA1AI

SLC26A9

XPR1

FLVCR1

SLC35F3

SLC16A3
SLC26A11

SLC6A19

SLC22A23
SLL9A3

SLC9A3

SLC25A19
SLC16A6
SLC45A1

SLC45A1
LETM2

SLCLAS

SLC 30 A 3

SLCBSE2A

SLC25A12
SLC35E2B

SLCTAAO

SLC47A2

SLCOOA

SLCBAT

SLLSATO
SLCIA3

SLC38A11

SLC43A2

SLCAA3

SLC39A10

SLC25A35

SLC35ES

SLC22AT

SLCSAT

SLC12AT

SLC12A5

SLCGAB

SLC26A3
SLCTA1

SLCTAT
SLC12AS

SLCA4AS

SLC5A4

SLC13A4

RHCG

SLC22A5

SLCO2A

SLCB7A

SLCGAG

SLCAA4

SLCBAT

SLCGA11
SLCAA7

SLCAAT
SLCLAG

SLCLAG

SLC4AA3

SLCO1B

SLC6A14

SLCAA11

SLCSA11

SLCBOA2

SLC45A2

SLC16A

SLC22AA

SLC51B

SLC14A

SLC36A

SLC35F2

SLC16AS

SLC29A4

SLC25A14

NLC1

SLC15A2

SLC46A

LCTAA

SLCTA4

SLCO4CI

SLC26A6
SLCAA2
SLC3562
SLC25A53

SLCEAA

SLCGAG
SLCI6AIA

SLC12AE

SLC10A

SLC16A4
SLC22A10

SLC22A10

SLC22A12

SLC22A31

SLCGA9

SLC22A15

SLC5A2

SLCBBAS

SLCB8AG

SLCTAA

NIPAT

SLC22AI

SLCTAG

SLC35E

UCP3

SLC25A

SLCBвA

SLCA1A

SLC39A

SLC39A3

SLC25A4

SLC38A8

\begin{tabular}{l} 
SLCSBA \\
SLCAA14 \\
SLC10A6 \\
SLC25A51 \\
\hline
\end{tabular} 
Table S3 The associations of the 31 DESLCs with the clinical features

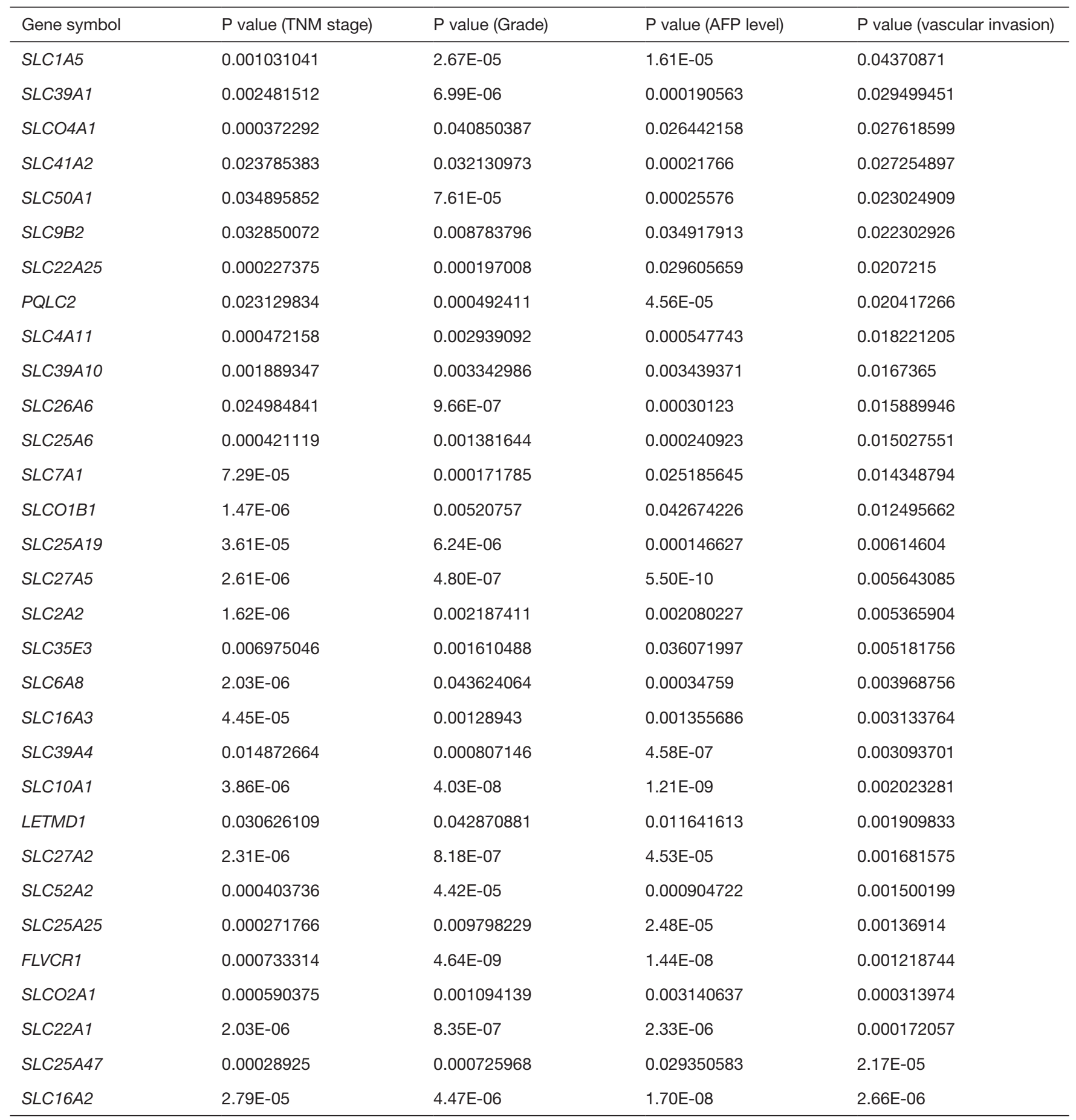


Table S4 The associations between the clusters and the clinical or genetic features

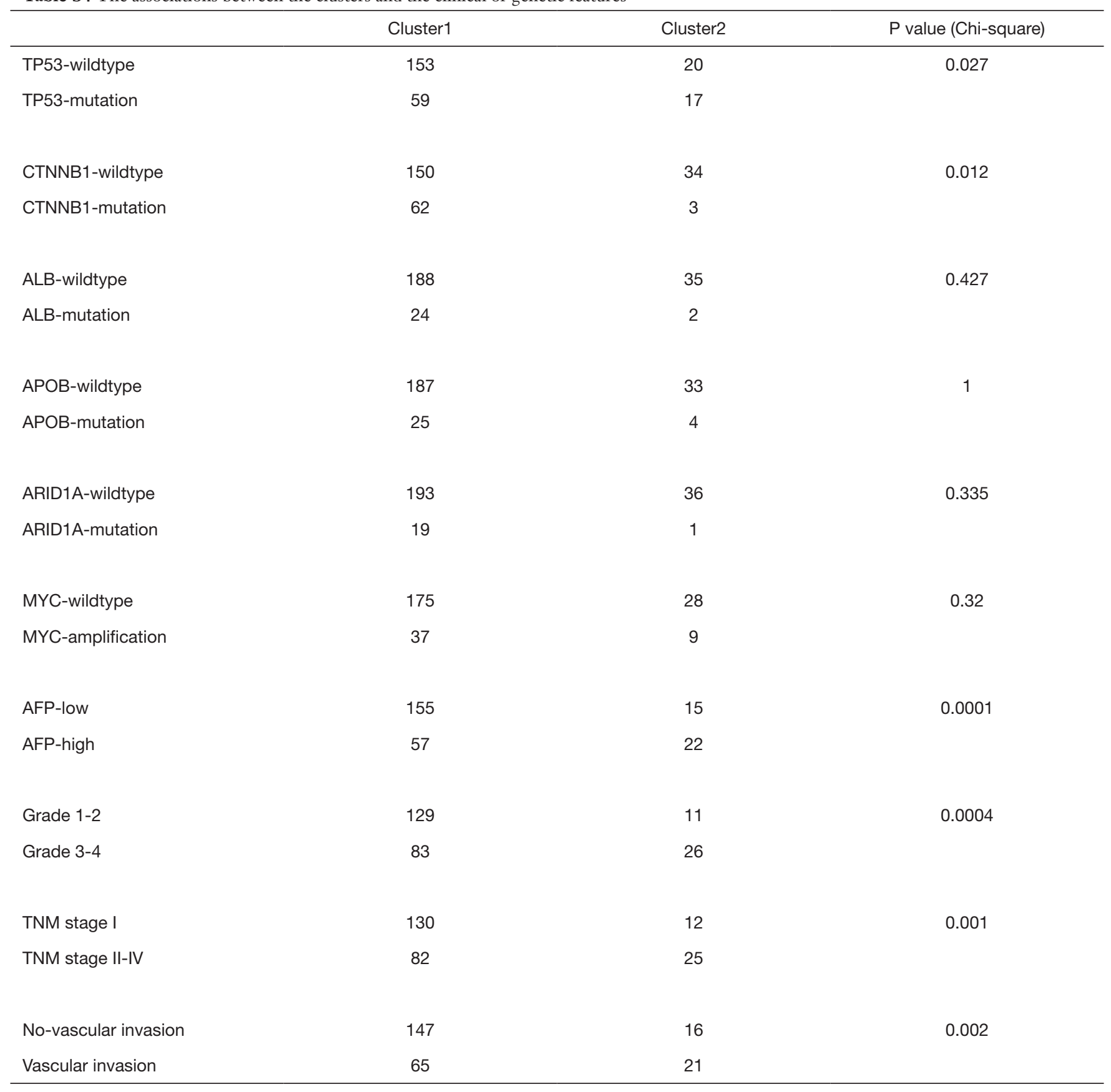

